OPEN ACCESS

Edited by:

Anthony Christopher Vernon, King's College London, United Kingdom

Reviewed by: Carmine M. Pariante, King's College London, United Kingdom Stefano Berto,

University of Texas Southwestern Medical Center, United States

${ }^{*}$ Correspondence: Silvia Sánchez-Ramón ssramon@salud.madrid.org

Specialty section: This article was submitted to Molecular Psychiatry, a section of the journal Frontiers in Psychiatry

Received: 10 March 2020 Accepted: 04 September 2020 Published: 29 September 2020

Citation:

Sánchez-Ramón S and Faure F (2020)

Self and the Brain. The Immune Metaphor.

Front. Psychiatry 11:540676. doi: 10.3389/fpsyt.2020.540676

\section{Self and the Brain. The Immune Metaphor}

\author{
Silvia Sánchez-Ramón ${ }^{1,2^{*}}$ and Florence Faure $^{3}$ \\ 1 Department of Clinical Immunology, IML and IdISSC, Hospital Clínico San Carlos, Madrid, Spain, ${ }^{2}$ Department of \\ Immunology, ENT and Ophthalmology, Complutense University School of Medicine, Madrid, Spain, ${ }^{3}$ INSERM U932, PSL \\ Research University, Institut Curie, Paris, France
}

One of the fundamental questions in neuroscience is how brain activity relates to conscious experience. Even though self-consciousness is considered an emergent property of the brain network, a quantum physics-based theory assigns a momentum of consciousness to the single neuron level. In this work, we present a brain self theory from an evolutionary biological perspective by analogy with the immune self. In this scheme, perinatal reactivity to self inputs would guide the selection of neocortical neurons within the subplate, similarly to $T$ lymphocytes in the thymus. Such self-driven neuronal selection would enable effective discrimination of external inputs and avoid harmful "autoreactive" responses. Multiple experimental and clinical evidences for this model are provided. Based on this self tenet, we outline the postulates of the so-called autophrenic diseases, to then make the case for schizophrenia, an archetypic disease with rupture of the self. Implications of this model are discussed, along with potential experimental verification.

Keywords: neurologic self, immune self, neurogenesis, schizophrenia, autophrenic disease

\section{A JOURNEY FROM CONSCIOUSNESS TO CELL BIOLOGY}

Since the philosopher David Chalmers raised "the hard problem of consciousness" (1), referring to the interrelationship between brain activity and the content of conscious subjective experience, there have been numerous attempts to explain consciousness from a scientific approach. Innovative approaches have arisen from neurophenomenology (2), functional neuroimaging tools (3) and from cognitive and evolutionary psychology (4). Through most of the Western science, consciousness has been considered as an emergent brain process: the result of the integration of either global neural synchronization $(5,6)$, or of many asynchronic microconsciousness (7), whereas a role for the single neuron unit is left aside. However, based on quantum physics, it has been hypothesized a cell-based theory that located the momentum of consciousness within the neuron microtubules $(8,9)$. Yet, it has been largely unexamined the question of a cell-based consciousness from a biological viewpoint underlying microcircuitries (10).

Here, we explore the gap between self-consciousness and neural activity from an evolutionary biological perspective (11). There is a fundamental ontological assumption that the central nervous system (CNS) evolved along the immune system (IS) by natural selection to better define individuals' identity and interactions (12-14). We formulated the hypothesis that the distinction of self/nonself (internal/external) inputs by the brain has a cell basis similar to the immune self. The immune self/ nonself recognition enables immune effective function without jeopardizing tissue integrity (15). We 
inferred this self brain hypothesis by analogy of basic principles between these two complex network systems, although through divergent processes and scales. Contemporary systems biology aims to understand the rules governing dynamic regulatory networks across different scales (genes, RNA, proteins, cells, systems, metasystems, organism). Self-organization underlies a generic property of many complex networks and of gene regulatory networks in particular, which control cell ontogeny $(16,17)$.

Comprehensive understanding of immune physiology has often been anticipated by clinical immunopathology. For instance, autoimmune diseases in vertebrates are induced by failure or imbalance in recognition of the tissues of the body itself. However, autoimmunity or low level self-reactivity is essential for normal immune response. Within the CNS, an inappropriate reactivity to self inner signals would risk of an "horror autotoxicus"1 (18) severely interfering with any cortical sensory, motor, and cognitive processes and therefore with consciousness. Inappropriate reactivity to self may characterize what we have called autophrenic diseases [from Greek $\alpha \mu \tau$ o-

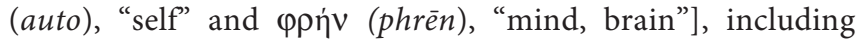
epilepsy or schizophrenia (SZ). According to this novel view, epilepsy may represent excessive reactivity to self ${ }^{2}$, defined by sudden firing of a subset of neocortical neurons that causes unrealistic sensations (visual, olfactory, gustatory, auditory), involuntary jerking and altered self-consciousness (19). In the case of SZ, it would occur a dysfunction of subsets of neocortical neurons that discriminate self body signals as nonself, resulting in the rupture of the psychoneurological self $(20,21)$. Indeed, false fragmented perceptions are lived as a real self/nonself conflict and severely affect the patient's awareness. These pathologies might suggest a role for the self at the brain subscale microcircuitries or even at the cellular level.

Immune self/nonself discrimination is instructed mainly during embryogenesis and early in life, through selection processes resulting in massive cell death that ensures efficient and highly specific response and eliminate potential highly autoreactive lymphocytes. The principles guiding brain cortex functional organization during embryonic neurogenesis have not been fully investigated. If, following our argument, that were the case that a self principle governs brain function, such instruction in self should be developed during neurogenesis. Based on theoretical grounds and biological observations, we hypothesized that self-driven selection processes based on neuron connectivity occur within the brain cortex (11). In this article, to translate our hypothesis into a model, we firstly recapitulate well-established immune principles to hypothesize similar brain solutions at the system level in a unified evolutionary account; and secondly, we present a body of arguments to prove the plausibility of the hypothesis based on clinical and experimental observations. We then expand the

\footnotetext{
${ }^{1}$ Paul Ehrlich proposed the term "horror autotoxicus" to designate the unwillingness of the organism to the production of autoantibodies "amboceptors directed against its own tissues".

${ }^{2}$ Hereinafter "self" alludes to biologic self (at cell level) although otherwise specified.
}

analogy to outline the postulates for autophrenic diseases by extrapolating the postulates that define autoimmune diseases.

\section{Evolutionary Immune-Based Model for Brain Memory}

The IS comprises at least two inextricably intertwined orders of recognition and memory that were developed through evolution: innate and adaptive immunity, the latter initially acquired in jawed vertebrates. Innate immunity displays a broad and pseudospecific quick and transitory response to stimuli (trained memory), essential for the survival of the organism as it is the only immunity present in invertebrates and plants. Adaptive immunity serves a highly specific and controlled response to a given antigen, and accounts for a long-term memory that, upon an ulterior rendezvous with this antigen is translated in a more accelerated, focused, and heightened response (22). Adaptive specificity stems from a bewildering diversity of unique $\mathrm{T}$ and $\mathrm{B}$ cell receptors repertoire, representing thus a huge qualitative jump in fine specificity to a changing environment by increasing complexity and efficiency of the response. Interestingly, innate immune cells (antigen presenting cells) drive antigen-specific memory activation and clonal selection of T lymphocytes' pools.

We built on the similarity of this functional organization with the vertebrate CNS looking side by side innate/implicit and adaptive/explicit orders of recognition and memory (23). Through these analogies, our model challenged the traditional view of the hippocampus only ascribed to the explicit/adaptive memory by an innate/implicit memory structure and essential mediator to explicit (declarative) memory. Although the hippocampus and adjacent structures of the medial temporal lobe (MTL) share function homology to the mushroom bodies of invertebrates (such as insects and crustacea) (24-26) and are evolutionarily ancient brain regions, the involvement of MTL in short-term associative memory is recent $(27,28)$. Hence, MTL bridges both implicit and explicit processes, which is not a new idea, but in line with kinetic models (29), functional neuroimaging studies $(30,31)$, and processing-based models (32). Explicit/adaptive memory is founded in an overwhelming diversity of neocortical neurons spatially and dynamically arranged to cope with extremely precise recognition that enables high cognitive abilities. Similar to the IS, "innate" brain regions (MTL) guide the formation of long-term and highly accurate explicit/adaptive memories through specific synapses between ensembles of neurons (engrams) within the neocortex (33). The proposed classification of brain memory based on a singular immune standpoint opens up a broader evolutionary insight on the role of the MTL in implicit and explicit memory strategies of encoding, storage, and retrieval (23). For instance, the widely studied patient H.M., with extensive bilateral hippocampal lesion, is an eloquent case supporting that highly specific (explicit/adaptive) memory resides within the neocortex, and corroborates the psychological distinction between shortand long-term memory (34).

Our epistemological approach explores recognition and memory brain systems by an immune-based analogy to better align the functional network architecture within an evolutionary 
context. This standpoint has not been previously undertaken but to applying IS principles into artificial intelligence computation (for instance, data encryption and storage, intruders' detection and recall algorithms, information rate efficiency, machine learning, etc.) $(35,36)$. Depicting similarities in basic biological principles between the IS and the CNS does not mean diminishing their gross differences or pretending to tackle the self, which lies beyond the boundaries of cell biology. In this essay, we intend to offer a new perspective on how brain emergent properties may be constrained by cell biology.

\section{Instruction in Self: Thymopoiesis Meets Neurogenesis}

The IS and the CNS constitute two complex network systems in open exchange that sense and respond to the environment while preserving the identity and integrity of the organism (homeostasis). This information processing function requires the discrimination between a dynamic self (internal signals and symbiotic interactions) and nonself (i.e. the outer world and the other), property shaped through evolution up to a degree of high specificity (37). In addition to higher specificity and specialization, evolution has led to an everincreasing complexity at genetic, molecular and cellular regulatory interactions (complementarity, positive and negative feed-forward and feed-back loops) to determine overall response within each system. It can be asserted that the specificity of $\mathrm{T}$ lymphocytes and neocortical neurons as a whole delineate our identity (immunological and cognitive) as individuals, which is mostly acquired during embryogenesis and shortly after birth. Hence, each one of us is equipped with a unique repertoire of $\mathrm{T}$ lymphocytes and neocortical neurons $(38,39)$ to face the external world early in life. These cells will be thereafter selected by novel stimuli from the external world through connections with innate cells/structures, triggering activation of cells ensembles; and the repertoire of lymphocytes and neurons will be then shaped according to external experience (40). The whole process underpins the extraordinary plasticity of these systems and the concept of individual history. Dealing with the notion of time is indeed an intricate issue of the IS and the CNS, which has equally fascinated physicists, biologists, and philosophers approaching the brain in particular. To put this temporal relationship in plain terms, these paradoxical "anticipatory" specific cells ( $\mathrm{T}$ lymphocytes, neocortical neurons) can be selected at any given moment by external cues to become "past" memory cells ensembles and then travel forward to "the present" -now- during recall, while being key to modifying the "future" behaviour of the organism (definition of cognition).

The anticipatory repertoire of T lymphocyte receptors (TCR) is generated by combinatorial gene rearrangement within the thymus (thymopoiesis). T-lymphocytes subsequently undergo a multi-step process of selection in response to self (41-43). Those T lymphocytes that weakly respond to self stimulus (antigen) happen to survive, while those that do not react with self die, (positive selection), and those that react too strongly with self are most of them eliminated (negative selection) or preserved as regulatory $\mathrm{T}$ lymphocytes $(44,45)$. Thus, the self principle governing thymopoiesis determines the future immune response. As Janeway's proverbial assertion dictates, "the immune system evolved to discriminate infectious non-self from noninfectious self" (43). Thus, autoimmunity (low level autoreactivity) is an inherent constituent of immune homeostasis, meaning that all peripheral $\mathrm{T}$ cells are self-reactive (46). The process described above is greatly simplified to focus on general mechanisms, and even if TCRs are highly specific, restriction of antigen presentation by the major histocompatibility complex (MHC) further increases the diversification of the individual response and is subjected to relative degeneracy.

How does the exquisite brain organization contribute to effective self/nonself recognition? And moreover, can we infer that there is a role for cognitive self at the neuron (engram) level? The underlying principles and mechanisms generating the functional specificity and diversity of neocortical neurons are far from being well known.

In a recent work, we suggested proceeding from an immune angle to address these questions and testing its validity or refutability. From this standpoint, we positioned the self as the axis of cortical neurogenesis (11), which would allow normal brain functioning and prevent costly autoreactivity. In parallel with the immune "logic", we sustained that the brain evolved to discriminate perceptible non-self from non-perceptible self. Neocortical neurons selection would be guided according to the degree of self recognition, by which too low and too high self-reactive neurons would undergo programmed cell death. Such self-driven neuronal selection would remove neurons exhibiting none or excessive reactivity to self signals. As a result of this selection process, neurons exhibiting low selfreactivity would discriminate any novel external stimulus, fundamental for effective neuronal response, and tolerance induction in the neocortex (11). This would also mean that all neocortical neurons are somehow self-reactive. Interestingly, neocortical neurons are not only "perceptive" of the outer world but highly interconnected via associational projections to fulfil the needs of the organism by using internal and external information.

Neurogenesis is written in chemical and electromagnetic language, whose code has been explored from multiple perspectives. We present below a body of arguments to test the presented hypothesis based on experimental and clinical observations, notwithstanding the obvious limitations derived from current gaps in knowledge and to methodological barriers ${ }^{3}$.

i. Internal cues during neocortical neurogenesis primarily instruct neuronal selection:

-The most prevailing model for the development of neural circuits (Hebbian plasticity) states that synaptic connections are strengthened by correlated activity between pre- and postsynaptic neurons, while weakened by uncorrelated activity or lack of activity. Neural activity-dependent regulation is involved in cell type specification, dendritic branching, synaptic maturation and learning and memory through a complex program of gene regulation (47). However, this theory does not explain to date whether and how activity-dependent

\footnotetext{
${ }^{3}$ References to the original papers will be made when these provide essential contribution to the discussion.
} 
mechanisms sort out neurons during neurogenesis. Two sequential waves of programmed cell death (PCD) regulated each by two distinct gene programs occur at the cortex subplate during embryogenesis and early postnatal life: a first wave at ventricular (VZ) and subventricular zones (SVZ) that purges up to $70 \%$ of progenitor cells showing spontaneous voltagedependent activity evolving to synchronized small networks; and a second wave that further selects around $\approx 30 \%$ of mature neurons at postmitotic zones once coherent neural circuits with thalamocortical and cortico-cortical connections have been established [reviewed in (48)]. According to our model, to delete neurons by internal (self)-reactivity criteria, which goes beyond neuron-quality control, the whole process evokes striking similarity with the two independent PCD waves of positive and negative selection of lymphocytes during thymopoiesis in: developmental timing (embryonic and postnatal); stepwise functional segregation according to cell activity (primarily of inactive progenitor cells and of synaptically-driven maturing neurons afterwards); cell specification and migration-maturation gradient; balanced specific excitatory and inhibitory cell subsets (49-52). We suggest that PCD purges primarily those neurons that do not show a reaction to internal self-signals and afterwards eliminates those that overreact to these internal signals (11).

-In vitro and in vivo findings support that endogenous spontaneous firing rates at the neocortex may guide PCD waves during neurogenesis $(53,54)$. It remains unclear whether this neural activity functions in an instructive or in a permissive way, that is, if there are specific patterns of neural activity leading the neuronal fate; or in the contrary, it is that the mere presence of neural activity is sufficient for the neuronal survival. In fact, it has been described a rich repertoire of organized spontaneous activity patterns within the neocortex intra utero and perinatally, whereby depolarization of transmembrane voltage potentials above a certain threshold affects neurons survival and network organization $(52,55-57)$. By analogy to the presentation of the wide array of body self antigens (ectopically) to lymphocytes during thymopoiesis for self instruction, we hypothesize that the neurogenesis harbors the huge cast of self electrical signals that will instruct and select by PCD the repertoire of neocortical neurons. If this is so, the search of promiscuous gene expression within the cortex of those self electrical signals to promote self tolerance-the equivalent to the autoimmune regulator (AIRE) gene by thymus stromawould be of great interest. We have not found experiments addressing specific-spike series or specific molecules within the SVZ/subplate in relation with differential neuronal sorting and circuit formation that may explain specific pattern-dependent regulation in neuronal segregation.

-Experimental models of cortical neurons xenotransplantation may shed light on the issue of a potential host self brain instruction. After single human pluripotent-stem cells (PSC)-derived cortical neurons xenotransplanted to the neonatal (P0/P1) mouse brain into the subplate, neurons integrated in the mouse cortex. Around $17 \%$ of transplanted single neurons matured and displayed responses to sensory stimuli that resembled those of the host neurons, stressing the specific nature of the circuitry. The authors suggested that the host brain provided not only permissive environment but also instructive cues regulating precise circuit formation (58). The host self instruction in human neurons seemed to be restricted during maturation at subplate by presynaptic partners (thalamus or cortex). The experiment was performed in neonatal mice, showing ulterior fine-tuning to external stimuli. By contrast, very limited synaptic integration results when the PSC-derived human cortical neurons were transplanted in bulk into the mouse cortex, which are less accesible to receive inputs from the host brain (59).

ii. The presence of specific excitatory (E) and inhibitory (I) neuron subsets within the neocortex assures homeostasis and functionality.

-A cortical organoids model from induced-PSC generates $\mathrm{E}$ and I neurons, i.e. glutamate and gamma-aminobutyric acid (GABA) neurons, respectively, which account for the generation and maintenance of oscillatory activity and synchronization of the network. Small-scale functional electrophysiological networks by these neurons subsets coordinate information flow resembling preterm neonatal brain activity (60). Inhibitory neurons act as a necessary "self-check" for excessive or prolonged responses by which the cortex precisely regulates functional effective connectivity. Impaired E/I balance is associated with several diseases, such as epilepsy and SZ.

-Stimulus-specific E and I assemblies have been described in the ferret primary visual cortex (61) and posterior parietal cortex of mice (62), pointing to selective inhibition by GABAergic neurons, similarly to antigen-specific regulatory $\mathrm{T}$ cells, controlling excessive responses and maintaining homeostasis and tolerance to self.

Recent work has shown that positive selection of I neurons (early postnatally) occurs and is coordinated by activitydependent connections to $\mathrm{E}$ neurons $(63,64)$ ). The generation of the combinatorial code of unique neuron-tag molecules, such as protocadherins (65), seems to regulate a critical window of PCD of cortical interneurons (66). Adequate balanced networks of I and $\mathrm{E}$ neurons is adjusted by consecutive waves of PCD $(48,63)$-. This phenomenon further supports the concept of neuron-specific selection by self $\mathrm{E}$ neurons, providing an evolutionary advantage for the rapid increase in pyramidal neurons in the primate lineage (63).

iii. Lesion studies can yield valuable information about the putative contributions of neural selection in utero to cortex functionality.

-Malformations of the cortical development (MCD) may be due to a broad array of disorders that disrupt the tightly spatiotemporally orchestrated process of neurogenesis (proliferation, migration, differentiation, synaptogenesis, apoptosis, synaptic pruning). MCD may affect the neuronal pool and connectivity of specific circuits, causing a wide spectrum of cognitive deficits, seizure disorders or neurospychiatric diseases, such as schizophrenia or autism (67). Depending on the time and degree of the neurodevelopmental 
insult, clinical onset can be delayed (latency) thanks to compensation mechanisms through E-I interactions or other plasticity mechanisms, or to the time lapse to acquire a task that relies upon the specific neurobiologic substrate. A main feature is thus the fine regional and functional specificity of the affected neocortical neurons, which translates into hyperexcitability (anti-self) interfering their related circuits.

-The self brain hypothesis can be fully integrated into the programmed changes described for differentiation and maturation sequences of cortical neurons during neurogenesis and also provides a new dimension to the whole biological process. Defects or interferences in these developmental changes would result in excess of specific autoreactive E neurons, contributing to the hyperexcitability and ultimately in epilepsy. The view we present here might add conceptually important elements to the understanding of epileptogenesis. Accumulative or dysbalanced action potential firing of individual autoreactive neurons may disrupt the ensemble of specific circuits. The earlier the insult, the more severe or intractable the disorder, with persistent deleterious effects despite the high plasticity of the immature brain $(68,69)$. Subtle alterations on electrical activity during neurogenesis affect neuronal segregation and connectivity, and can cause many forms of epilepsy. These observations may suggest that specific features of neural activity (rather than just the presence of neural activity) are important for the neuronal selection during neurogenesis, pointing to the hypothesis that endogenous specific neural activity is instructive for neuronal selection within the neocortex. This fact may also reflect the purging function of non-reactive or highly self-reactive neurons. Our theory may also provide the basis for therapeutically significant avenues of development. Currently available pharmacological treatments of epilepsy are mainly symptomatic, none is curative or preventive. Moreover, anticonvulsivants show suboptimal effectiveness with longterm detrimental neurologic effects. New functional tools based on specific cellular resolution biomarkers to identify the hyper-reactive E neurons populations may favour new therapeutical interventions to selectively block these circuits. Also, these neuronal resolution biomarkers could propel the dissection of specific circuits and hopefully the development of new drugs based on pathophysiological mechanisms.

-Gene lesions associated to control of apoptosis during neurogenesis underlie several types of MCD, resulting in epileptogenesis when not to perinatal lethality (70). Given that genes and epigenetic modifications regulating the survival of specific populations of neurons are now beginning to be elucidated, advances within the field will foster progress in understanding cortical neuron segregation and neural circuits during neurogenesis $(48,71)$.

-Murine models of MCD, in which targeted chemical and physical insults during early development within the SVZ induce pronounced cortical hyperexcitability and reproduce the pathological and clinical findings of congenital forms of epilepsy [reviewed in (72)]. Timing and location (region and layer) of the induced lesion are key to the MCD clinical expression, suggesting that specific alteration of neuronal seggregation processes lead to hyperexcitability and altered connectivity.

iv. Our hypothesis challenges the currently accepted alternative hypothesis of "instruction from external inputs":

-In the auditory system, the selection and wiring of neocortical neurons within central sensory areas precedes the formation and priming of sensory receptors, circuits that will be refined later on by external inputs $(73,74)$. Before hearing onset, the precise temporal pattern of spontaneous pre-hearing activity is crucial for the formation of precise tonotopy in the central auditory pathway, supporting the role of self-instruction orchestrated development.

-Extreme examples or experiments of nature, such as complete unimodal sensory deprivation or anophthalmia (bilateral congenital absence of eyes) may give relevant insight into this issue. The connections patterns of organization in the cortex visual areas in the absence of retinal waves and visual experience of anophthalmic patients are not significantly different from normal sighted individuals (75-77). This finding may suggest that the visual retinotopic architecture of the neocortex does not primarily depend on external sensory instruction, but that in utero neural activity primarily shapes functional properties of cortical networks $(75,77)$.

Therefore, from many directions we find support for the working hypothesis that self/nonself discrimination is the result of a biological process primarily instructed from early neurogenesis by host self signals, to build an extensive repertoire of neocortical neurons. In both the IS and the CNS, each post-selection repertoire would thus represent, respectively, a mirror image of the immune and neurological reality that we are able to sense and with which we can constantly interact (Figure 1). This primary neuronal repertoire and neural circuits will be secondarily refined by external inputs during development, an activity-dependent process that is plastic. The entire process would endow the brain with a cell basis for consciousness and hence self-consciousness.

\section{CONCEPTUAL, CLINICAL AND EXPERIMENTAL ARGUMENTS OF AUTOPHRENIC DISEASE. REVISITING SCHIZOPHRENIA}

In order to formulate a general scheme and a case example, we will firstly take advantage of the well-established criteria defining autoimmune disease $(\mathrm{AD})$ (78) to draw the principles of autophrenic disease in order to evaluate the self model in brain pathology (Table 1). Secondly, we will apply these principles to predict SZ pathophysiology. ADs are multifactorial conditions that result from the complex interplay of risk and protective factors, in which autoreactive $\mathrm{T}$ lymphocytes induce specific tissue damage or dysfunction. Intrinsic (genetic, epigenetic, endocrine, and psychoneurological), extrinsic (environmental), and stochastic factors induce cumulative effects that eventually 


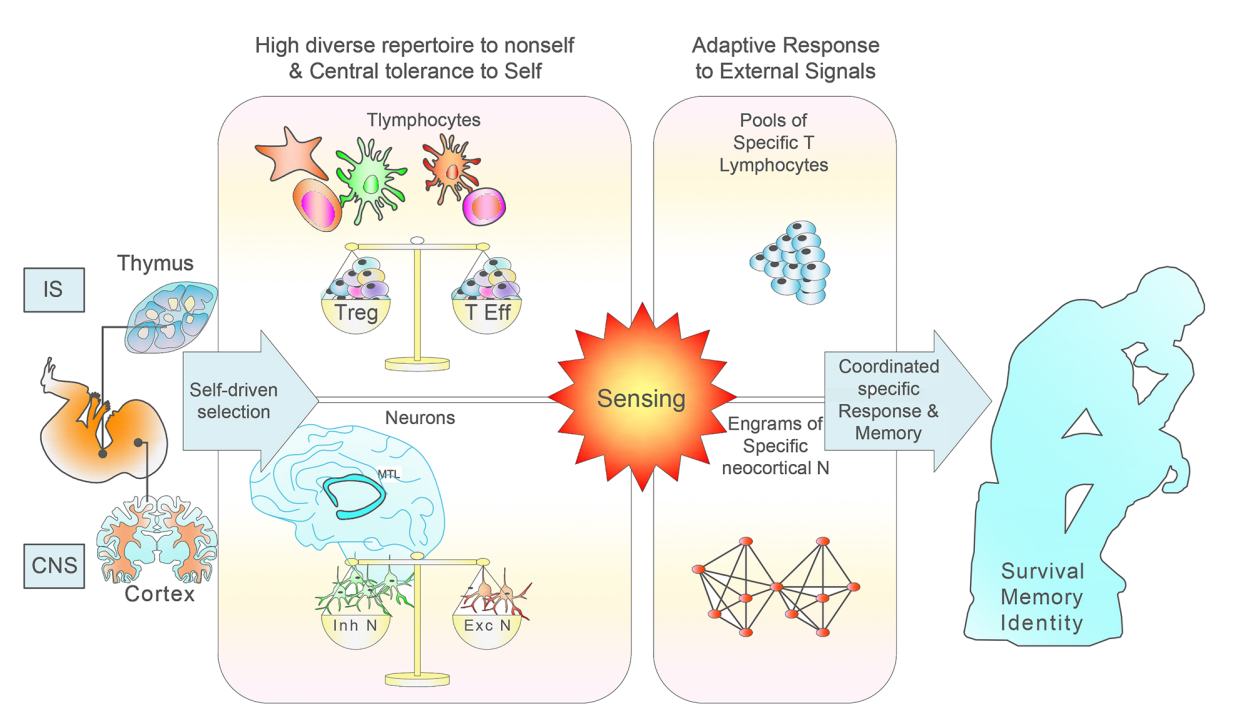

FIGURE 1 | Scheme of the normal IS and CNS development, maturation and functioning based on self education. During the generation of T lymphocytes in the thymus, it occurs a multi-step process of selection in which the great majority of highly self-reactive $T$ cells die, leading to an immunocompetent and self-tolerant pool of naïv T lymphocytes. Clonal deletion is incomplete, as many self-reactive T cells find their way to the periphery where they constitute a constant risk for the development of autoimmune disease. During neurogenesis, the effective removal of most autoreactive neurons relies on a coordinated multi-step selection, where most low reactive neurons to self would be able to discriminate external signals. A number of selection mechanisms is of fundamental relevance for neocortical neuron development, responsiveness to external signals and tolerance induction to internal signals. Matching of electrical signals through sequential layers allows combinatorial signals with increasing complexity in a unique and extremely precise way.

lead from health to illness, with a waving course. A key pathophysiological role of innate immunity in $\mathrm{AD}$ has been unveiled (98). An interesting phenomenon is how disparate $\mathrm{AD}$ share immune mediators (autoimmune tautology) (99), while differ in the target tissue or organ, highlighting that specificity is given by the antigen-specific autoreactive $\mathrm{T}$ lymphocytes. A proinflammatory/anti-inflammatory cytokine imbalance favours differentiation and amplification of these target-specific autoreactive $\mathrm{T}$ lymphocytes.

On the basis of this proposed neurobiological self model, the nomenclature autophrenic disease designates complex multifactorial diseases in genetically susceptible individuals, modulated by endocrine and immunological factors, as well as psychological events in life (11). They define excessive or aberrant responses of autoreactive excitatory cortical neurons to specific endogenous neural inputs and/or defective inhibitory neurons, which disrupt certain cortical brain structures and functions (Figure 2). Similarly to autoimmune tautology, a cortical E-to-I neurotransmitter imbalance characterizes autophrenic diseases. By analogy with autoimmune pathogenesis, evolutionarily ancient brain structures (in particular the limbic system) would be expected to play a primary role. To complicate the scenario even further, autophrenic disease may be triggered by autoimmunity, as autoreactive T lymphocytes can target specific neurons' subsets $(92,93)$.

$\mathrm{SZ}$ is a prevalent mental disease characterized by a severe and disabling course in which the rupture of the psychic self is nuclear to the disease (100). Endocrine factors, such as male predominance, clinical onset in adolescence or early adulthood, and worsening at postpartum may play a part, coincidentally with cognitive maturation of the prefrontal (PFC) and parietal cortices $(101,102)$. Foetal and early childhood immune priming, such as maternal infection or active brain inflammation, are strongly associated with susceptibility to disease (103). Cumulative evidence across different experimental approaches (copy variant numbers, rare and de novo variants, genome wide association analysis, transcriptome and 3D genome structures) has restored its original conception as a neurodevelopmental disease, which stretches the way back to neurogenesis during embryonic development $(88,104,105)$. SZ shows strong heritability estimated from twin studies of $79 \%$ (106, 107), while is highly polygenic with very low individual impact. Main mutations involve synaptic connectivity and chromatin remodelling (108). Transcriptome analyses of epigenetic regulated genome have revealed specific cell-type-dysregulation in the frontal lobe of SZ patients (109-111). In addition, a role of activated microglia during neurogenesis that affect neuronal segregation and connectivity has been hypothesized $(112,113)$. It is postulated that $\mathrm{SZ}$ is a heterogeneous large scale dysconnectivity syndrome (114). According to age of onset, SZ has been classified in a rare but severe childhood form with widespread cognitive impairment; and an early adult form with predominantly PFC-related verbal and executive abilities decline (115). Hallucinations and passivity phenomena (delusions of alien control), with disrupted discrimination between the external and internal inputs, are cardinal to SZ. In particular, auditory (audible thoughts, voices arguing and commenting about the patient in third person), visual and cenesthesic hallucinations are common (first rank symptoms). Overactivity in primary and secondary sensory areas seem to be 
TABLE 1 | Model of autoimmune disease criteria proposed by Rose and Bona based on 1957 Witebsky's postulates (78).

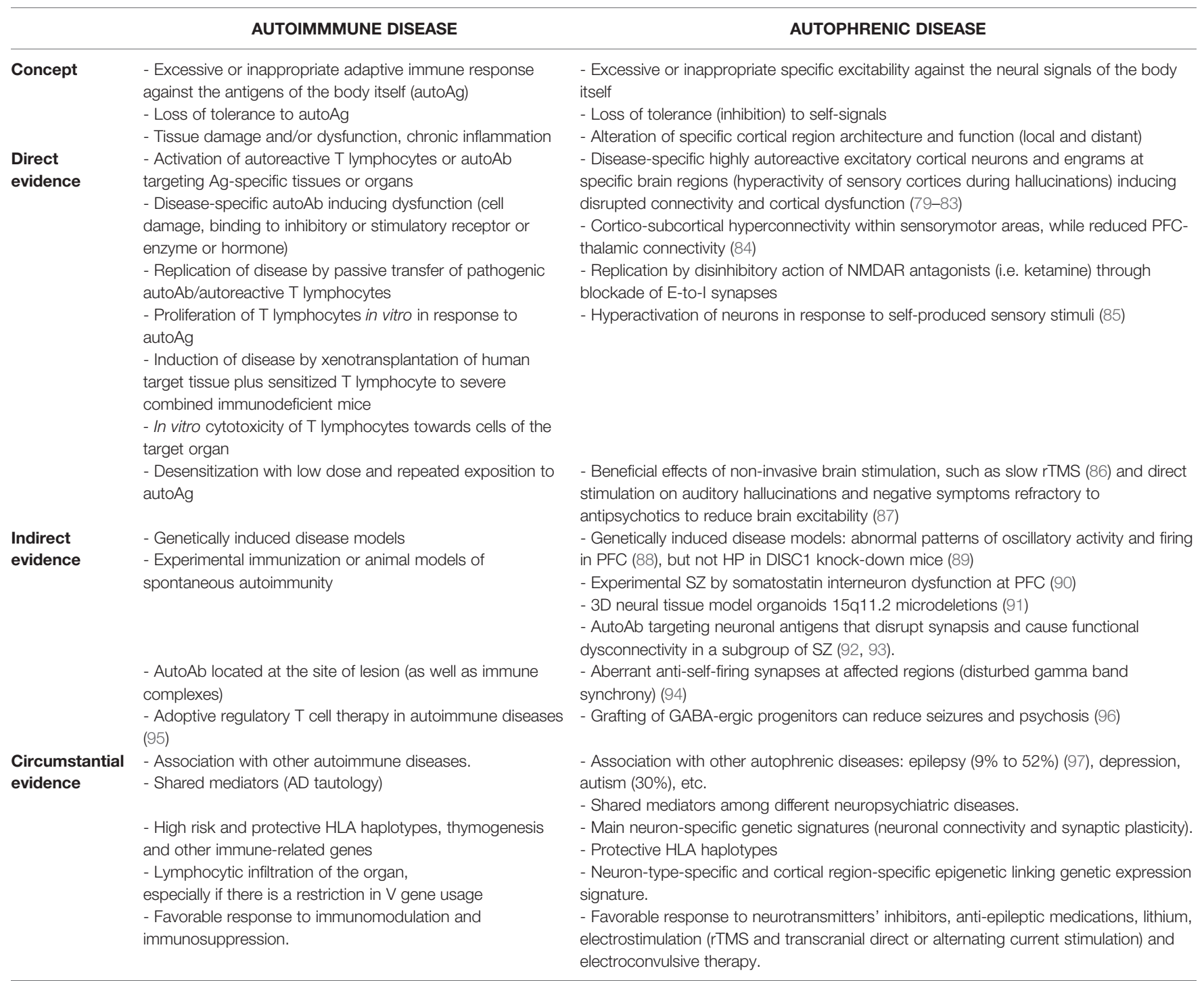

Ab, antibody; Ag, antigen, DISC1, disrupted in schizophrenia; HLA, human leukocyte antigen; E-to-I, Excitatory-to-Inhibitory; HP, hippocampus; NMDA-R, N-Methyl-D-Aspartate receptors; PCF, prefrontal cortex; rTMS, repetitive transcranial magnetic stimulation.

Autoimmune diseases in strict sense must fulfil at least three criteria of direct evidence and most of those of indirect and circumstantial evidence. We show autophrenic disease postulates in parallel and specifics applied to the case of schizophrenia.

involved in aberrant gamma oscillations to endogenous inputs from pyramidal neurons (83) despite absence of actual external stimuli (81, 82, 85). Some authors have explained this aberrant neuronal firing to inner signals in terms of feed-back loops and distant connectivity involvement with excessive expectation, by which altered recognition of endogenous signals were misattributed as coming from an external source (79). Experimental evidence suggests that SZ patients show similar or even outperform healthy controls on visual discrimination skills. More difficult is to explain negative symptoms (e.g., alexithymia) in terms of self, which might be understood by local and long-scale brain connectivity disturbances $(80,114)$.

The E/I imbalance is considered key for SZ pathophysiology (116). However, the non-uniform distribution of the E/I imbalance-such as distinct hyper-o hypodopaminergic (117) and hyper- or hypogabaergic brain regions and cortex areas (118) - suggests their secondary role in SZ pathophysiology. This uneven distribution dampens the therapeutic efficacy of current drugs. A profound defect in inhibitory GABAergic interneurons has long been established as the most common finding (119). GABAergic defect is region-specific, with decreased expression of the neurotransmitter GABA, decreased ability to generate gamma oscillations, decreased GABA receptors and low inhibitory neuron markers at the basal ganglia, the visual cortex and in the cerebrospinal fluid (120). In contrast, GABA is increased at PFC in unmedicated patients $(188,121)$. Excessive dopamine and glutamate (due to hypofunctioning N-methyl-Daspartate receptors) within the hippocampus and striatum (79, 122), account for overactivity of the primary and secondary sensory areas that induce misperceptions, while both 


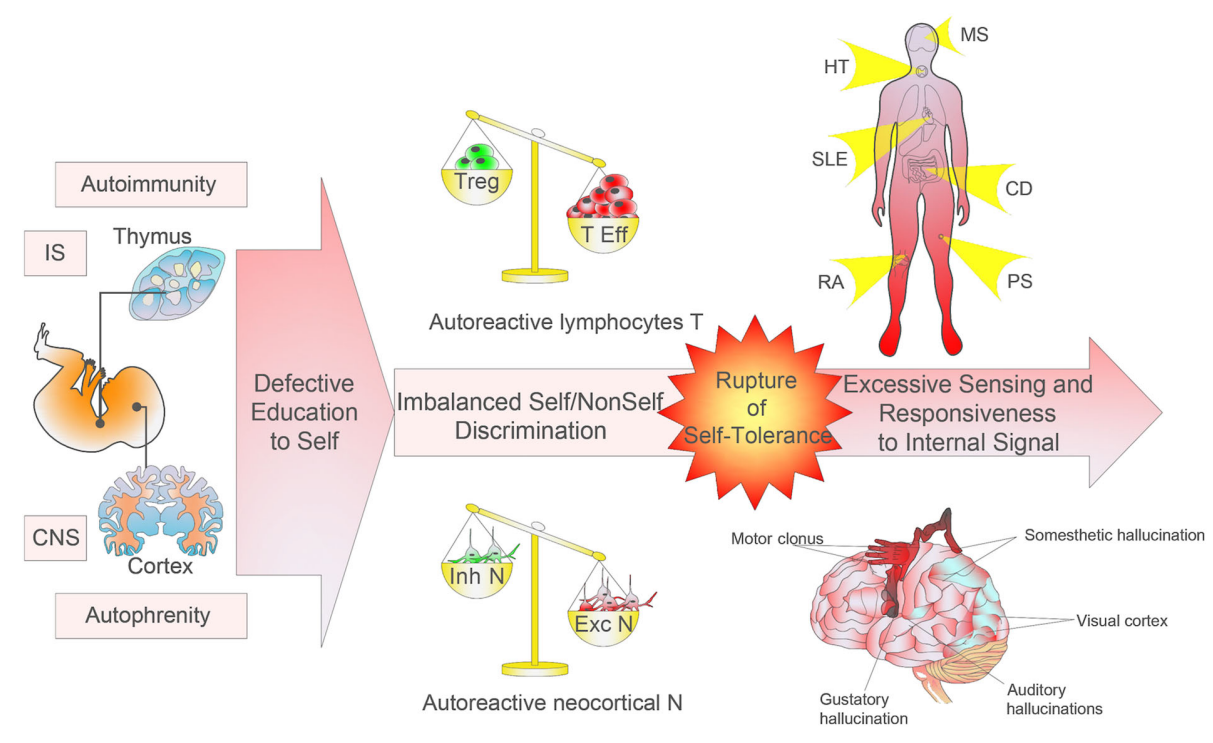

FIGURE 2 | Imbalanced self/non-self education and discrimination. The research on monogenic autoimmune syndromes has shown the relevance of mutations harbored by defined proteins of thymic selection processes and the development and activation of regulatory $T$ cells. Environmental factors operating in a genetically susceptible host may trigger or exacerbate the pathological autoimmune process, induce mutations in genes coding for immunoregulatory factors, or modify immune tolerance or regulatory and immune effector pathways. Autoimmune diseases can affect any site and any organ system in the body. In parallel, monogenic autophrenic diseases are related to protein mutations involved in cortical neurogenesis, synaptic plasticity and connectivity, and chromatin remodelling in specific cortical areas or functions, which can affect any body's function. The neocortex shows an extremely fine topography and plays essential functions of sensory, motor, and cognitive processes. The autophrenic diseases so conceived may provide a better understanding of the complex neurobiology of neurodevelopmental disorders. Although the autoimmune/autophrenic diseases affect specifically adaptive/explicit recognition, the pathophysiology is induced by alteration in evolutionarily more ancient cells constraining adaptive response (i.e. antigen presenting cells and the limbic system, respectively).

neurotransmitters are decreased at brain cortex. Conventional therapies have been directed to modulate the E/I balance, with partial effectiveness and potentially severe side-effects.

We suggest that E/I imbalance arises from dysregulation between self-specific excitatory and inhibitory neurons (or synapses) at circumscribed cortical areas. Involvement of the cortical areas responsible for specific recognition and explicit memory (primary and secondary sensory areas) might underscore a perceptual basis of self-consciousness. Thus, the dysregulation between self/nonself-reactive neurons would be the key event in the autophrenic disease, with subsequent local E/I imbalance and altered connectivity processing and metacognition. In this context, our theory advocates for the identification through single-cell resolution biomarkers of specific hyper-reactive neurons and the ensemble of their circuits as a new means for better understanding these diseases and for the exploitation of more specific blocking strategies. Alternativelly, targeting specifically GABA inhibitory neurons at the former circuits might be effective to control hyperexcitability. Promising strategies targeting specific cell types are being advanced by calcium imaging (123). Selective optogenetic activation of individual cortical neurons can trigger relevant ensembles and modify behavioural responses in mice, supporting a causal link between the cortical neuron (self-reactive neuron in our view) and learned behavior $(124,125)$. In other order of strategy, a psychodynamic approach would be interpreted as a means to "desensibilize" autoreactive neurons by modifying the context of presentation of the input from MTL or basal ganglia.

\section{DISCUSSION AND FUTURE PERSPECTIVES}

The IS and the CNS are genuine self-referential systems. The analogies between the two systems for cell recognition and memory strategies may support that general principles are operating. In the IS, discrimination between self and nonself is based on certain criteria of reactivity to self (46). Autoimmunity is an implicit constituent of cell immune homeostasis. Its deregulation may lead to autoimmune disease. In this work, we present a cell brain self theory from an evolutionary biological perspective by analogy with the immune self. At the cell level, we postulate that the extreme precision of recognition and association that enables the brain to perceive, memorize, anticipate, and to act is primarily instructed by reactivity to self during early life, when main neocortical structures and circuitries are organized. The implicit assumption of the biologic brain self theory is that autophrenity is consubstantial to brain physiology and homeostasis. Early life individual environmental exposure is key to refine the functional structure by means of a trade-off between chance (contingency) and necessity (adaptation). As for autoimmunity, the concept of autophrenity extends to self-regulation within the network. 
The actual role of the immune self/nonself principle to determine the outcome of the immune response is finely tuned to a spatio-temporal dimension. The response will depend not only in the antigen per se but in anatomic location (site-specific) and contextualization of the immune challenge (126). To illustrate the point made, if any antigen is delivered, how this antigen will be seen by the IS will be tuned by intrinsic (quantity, duration of exposition, location, etc.) and extrinsic factors that will differentially impact the response (effector against tolerant). Among the intrinsic factors, the valence, perceived at the system level as danger/reward or as discontinuity (surprise)/continuity (127), is key to the outcome and mainly driven by innate immunity. Likewise, the response of the CNS is not only determined by the stimulus per se but strongly conditioned by the context of presentation through innate structures (mainly the limbic system) as a danger/reward valence, by evolutionarily more sophisticated feelings (128) and by previous experience (129). This valence modulation has impressive therapeutic potential and is being exploited in strategies as, for instance, desensitization therapies in allergic diseases and by cognitive-behavioural psychotherapies (130), respectively.

The structure of cognition is metaphorical, built on pattern recognition at different scales (13). We endeavoured to explore the neurologic self as a metaphor of the immune self at the cellular level, which provides grounds for understanding complexity from another biological system logic. Through this immune analogy, our hypothesis provides a potential guiding principle, which may add both biologically and, likely, therapeutically significant avenues for development. The exploration by this approach of cortical neurogenesis might offer a bottom-up explanation of the system functioning as a whole, and a new insight into some neuropsychiatric diseases.

The present work addresses the autophrenic disease by analogy with autoimmune disease. Other approaches, such as computational phylogenetic analysis of homologue genes, which code for the receptor pathways of neocortical neurons across species, could add complementary experimental verification of the theory, which could trace the plausible evolutionary sequence. This theory constitutes the basis for current ongoing work.

SZ semiotics is already shifting to link mental phenomena with underlying neurobiological mechanisms $(131,132)$ given the overlap among psychiatric manifestations and diseases. As long as current theories about etiologically complex illnesses like SZ remain open, we hope our theory will help to change SZ understanding. Following this reasoning, our theory points at

\section{REFERENCES}

1. Chalmers DJ. Facing up the Problem of Consciousness. J Consciousness Stud (1995) 2:200-19. doi: 10.1093/acprof:oso/9780195311105.003.0001

2. Varela FJ. Neurophenomenology: a methodological remedy for the hard problem. J Consciousness Stud (1996) 3(4):330-49.

3. Dell'Italia J, Johnson MA, Vespa PM, Monti MM. Network Analysis in Disorders of Consciousness: Four Problems and One Proposed Solution (Exponential Random Graph Models). Front Neurol (2018) 9:439. doi: 10.3389/fneur.2018.00439

4. Mashour GA, Alkire MT. Evolution of consciousness: phylogeny, ontogeny, and emergence from general anesthesia. Proc Natl Acad Sci USA (2013) 110 Suppl 2:10357-64. doi: 10.1073/pnas.1301188110 aberrant anti-self neuronal responses behind anti-self neuropsychiatric disorders in a more meaningful dimension from a biological point of view. The brain self theory opens a new conceptual reflection on the gap between biological and conscious self. In his incompleteness theorem, Kurt Gödel decried the incapability for any formal system to be proven or disproven from within the system. Given the inherent limitations to consistently approach the functioning of the brain from brain logic, our inferential perspective from an immune metaphor would argue for its consistency. The brain self theory is beheld by a number of biological, experimental, and clinical findings in SZ that deserve further investigation. Typical positive SZ symptoms like hallucinations may help to better understand how excessive self-reactive excitatory neuronal activity of the neocortex may compromise the discrimination between the external world and internal experience and so alter the structure and connectivity of affected areas and distant circuits.

We expect that an understanding of neurobiology in terms of self at a wide-system functioning will open new targeted therapeutic strategies in disparate anti-self brain diseases and hopefully inspire further investigation.

\section{AUTHOR CONTRIBUTIONS}

SS-R wrote the first draft. SS-R and FF have equally contributed to the conception, design, critical revision, and final approval of the manuscript. SS-R and FF agree to be accountable for all aspects of the work in ensuring that questions related to the accuracy or integrity of any part of the work are appropriately investigated and resolved.

\section{ACKNOWLEDGMENTS}

This work was presented in part by SS-R at the meeting "Psychoses: Révolution des concepts, vers quelle évolution des pratiques?" October 19th, 2018, Paris. The authors gratefully acknowledge the generous aid, encouragement and suggestions of Alfred I Tauber. We are immensely grateful to Pablo Sáez, Ángel L Corbí and Jaime Adán for their unvaluable aid and criticism. We thank Marina Lorne for figure artwork and Antonio Castillo Algarra for his precise and careful English revision.

5. Dehaene S, Changeux JP. Experimental and theoretical approaches to conscious processing. Neuron (2011) 70(2):200-27. doi: 10.1016/j.neuron. 2011.03.018

6. Tononi G, Boly M, Massimini M, Koch C. Integrated information theory: from consciousness to its physical substrate. Nat Rev Neurosci (2016) 17 (7):450-61. doi: 10.1038/nrn.2016.44

7. Zeki S. The disunity of consciousness. Prog Brain Res (2008) 168:11-8. doi: 10.1016/S0079-6123(07)68002-9

8. Hameroff S, Penrose R. Consciousness in the universe: a review of the 'Orch OR' theory. Phys Life Rev (2014) 11(1):39-78. doi: 10.1016/j.plrev.2013. 08.002

9. Penrose R. The emperor's new mind: concerning computers, minds, and the laws of physics. Oxford, UK: Oxford University Press (1989). 
10. Edelman G. The Remembered Present: A Biological Theory of Consciousness. New York, UK: Basic Books (1989).

11. Sánchez-Ramón S, Faure F. The Thymus/Neocortex Hypothesis of the Brain: A Cell Basis for Recognition and Instruction of Self. Front Cell Neurosci (2017) 11:340. doi: 10.3389/fncel.2017.00340

12. Varela FJ, Coutinho A, Dupire B, Vaz NN. Cognitive networks: Immune, neural, and otherwise. In: Perelson AS, editor. Theoretical Immunology, Part II SFI Series on the Science of Complexity III. Redwood City: Addison-Wesley (1988). p. 359-75.

13. Edelman GM. Second nature: brain science and human knowledge. Yale University Press: London, UK (2006).

14. Kioussis D, Pachnis V. Immune and nervous systems: more than just a superficial similarity? Immunity (2009) 31(5):705-10. doi: 10.1016/j.immuni. 2009.09.009

15. Miller JFAP. The function of the thymus and its impact on modern medicine. Science (2020) 369(6503):eaba2429. doi: 10.1126/science.aba2429

16. Bornholdt S, Kauffman S. Ensembles, dynamics, and cell types: Revisiting the statistical mechanics perspective on cellular regulation. J Theor Biol (2019) 467:15-22. doi: 10.1016/j.jtbi.2019.01.036

17. Barabasi AL, Albert R. Emergence of scaling in random networks. Science (1999) 286(5439):509-12. doi: 10.1126/science.286.5439.509

18. Ehrlich P. Die Schutzstoffe des Blutes. Deutsche medizinische Wochenschrift (1901) 27:865-867, 888-891 und 913-916..

19. Chang BS, Lowenstein DH. Epilepsy. N Engl J Med (2003) 349(13):1257-66. doi: 10.1056/NEJMra022308

20. Nelson B, Thompson A, Yung AR. Basic self-disturbance predicts psychosis onset in the ultra high risk for psychosis "prodromal" population. Schizophr Bull (2012) 38(6):1277-87. doi: 10.1093/schbul/sbs007

21. Ebisch SJH, Aleman A. The fragmented self: imbalance between intrinsic and extrinsic self-networks in psychotic disorders. Lancet Psychiatry (2016) 3(8):784-90. doi: 10.1016/S2215-0366(16)00045-6

22. Farber DL, Netea MG, Radbruch A, Rajewsky K, Zinkernagel RM. Immunological memory: lessons from the past and a look to the future. Nat Rev Immunol (2016) 16(2):124-8. doi: 10.1038/nri.2016.13

23. Sánchez-Ramón S, Faure F. Through the Immune Looking Glass: A Model for Brain Memory Strategies. Front Cell Neurosci (2016) 10:17. doi: 10.3389/ fncel.2016.00017

24. McBride SM, Giuliani G, Choi C, Krause P, Correale D, Watson K, et al. Mushroom body ablation impairs short-term memory and long-term memory of courtship conditioning in Drosophila melanogaster. Neuron (1999) 24(4):967-77. doi: 10.1016/S0896-6273(00)81043-0

25. Mizunami M, Weibrecht JM, Strausfeld NJ. Mushroom bodies of the cockroach: their participation in place memory. J Comp Neurol (1998) 402(4):520-37. doi: 10.1002/(SICI) 1096-9861(19981228)402:4<520::AID-CNE6>3.0.CO;2-K

26. Strausfeld NJ, Wolff GH, Sayre ME. Mushroom body evolution demonstrates homology and divergence across Pancrustacea. Elife (2020) 9:e52411. doi: 10.7554/eLife.52411

27. Verfaellie M, Keane MM. Neuropsychological Investigations of Human Amnesia: Insights Into the Role of the Medial Temporal Lobes in Cognition. $J$ Int Neuropsychol Soc (2017) 23(9-10):732-40. doi: 10.1017/S1355617717000649

28. Sakaguchi Y, Sakurai Y. Left-right functional difference of the rat dorsal hippocampus for short-term memory and long-term memory. Behav Brain Res (2020) 382:112478. doi: 10.1016/j.bbr.2020.112478

29. Attardo A, Fitzgerald JE, Schnitzer MJ. Impermanence of dendritic spines in live adult CA1 hippocampus. Nature (2015) 523(7562):592-6. doi: 10.1038/ nature 14467

30. Jablonowski J, Taesler P, Fu Q, Rose M. Implicit acoustic sequence learning recruits the hippocampus. PloS One (2018) 13(12):e0209590. doi: 10.1371/ journal.pone.0209590

31. Schendan HE, Searl MM, Melrose RJ, Stern CE. An FMRI study of the role of the medial temporal lobe in implicit and explicit sequence learning. Neuron (2003) 37(6):1013-25. doi: 10.1016/S0896-6273(03)00123-5

32. Henke K. A model for memory systems based on processing modes rather than consciousness. Nat Rev Neurosci (2010) 11(7):523-32. doi: 10.1038/ nrn2850

33. Choi JH, Sim SE, Kim JI, Choi DI, Oh J, Ye S, et al. Interregional synaptic maps among engram cells underlie memory formation. Science (2018) 360 (6387):430-5. doi: 10.1126/science.aas9204
34. Squire LR, Wixted JT. The cognitive neuroscience of human memory since H.M. Annu Rev Neurosci (2011) 34:259-88. doi: 10.1146/annurev-neuro061010-113720

35. Farmer JD, Packard NH, Perelson AS. The immune system, adaptation, and machine learning. Physica D. Nonlinear Phenomena (1986) 22(1-3):187204. doi: 10.1016/0167-2789(86)90240-X

36. Huixin W, Duo M, He L. Analysis and simulation of the dynamic spectrum allocation based on parallel immune optimization in cognitive wireless networks. ScientificWorldJournal (2014) 2014:623670. doi: 10.1155/2014/ 623670

37. Swiatczak B, Tauber AI. Holoimmunity Revisited. Bioessays (2018) 40(11): e1800117. doi: 10.1002/bies.201800117

38. Chevee M, Brown SP. The development of local circuits in the neocortex: recent lessons from the mouse visual cortex. Curr Opin Neurobiol (2018) 53:103-9. doi: 10.1016/j.conb.2018.06.009

39. Roy M, Sorokina O, Skene N, Simonnet C, Mazzo F, Zwart R, et al. Proteomic analysis of postsynaptic proteins in regions of the human neocortex. Nat Neurosci (2018) 21(1):130-8. doi: 10.1038/s41593-017-0025-9

40. Olin A, Henckel E, Chen Y, Lakshmikanth T, Pou C, Mikes J, et al. Stereotypic Immune System Development in Newborn Children. Cell (2018) 174(5):1277-92 e14. doi: 10.1016/j.cell.2018.06.045

41. Alam SM, Travers PJ, Wung JL, Nasholds W, Redpath S, Jameson SC, et al. T-cell-receptor affinity and thymocyte positive selection. Nature (1996) 381 (6583):616-20. doi: 10.1038/381616a0

42. Krueger A, Zietara N, Lyszkiewicz M. T Cell Development by the Numbers. Trends Immunol (2017) 38(2):128-39. doi: 10.1016/j.it.2016.10.007

43. Janeway CAJr.. The immune system evolved to discriminate infectious nonself from noninfectious self. Immunol Today (1992) 13(1):11-6. doi: 10.1016/0167-5699(92)90198-G

44. Cheng M, Anderson MS. Thymic tolerance as a key brake on autoimmunity. Nat Immunol (2018) 19(7):659-64. doi: 10.1038/s41590-018-0128-9

45. Inglesfield S, Cosway EJ, Jenkinson WE, Anderson G. Rethinking Thymic Tolerance: Lessons from Mice. Trends Immunol (2019) 40(4):279-91. doi: 10.1016/j.it.2019.01.011

46. Tauber AI. Reconceiving autoimmunity: An overview. J Theor Biol (2015) 375:52-60. doi: 10.1016/j.jtbi.2014.05.029

47. Flavell SW, Greenberg ME. Signaling mechanisms linking neuronal activity to gene expression and plasticity of the nervous system. Annu Rev Neurosci (2008) 31:563-90. doi: 10.1146/annurev.neuro.31.060407.125631

48. Wong FK, Marín O. Developmental Cell Death in the Cerebral Cortex. Annu. Rev Cell Dev Biol (2019) 35:523-42. doi: 10.1146/annurev-cellbio100818-125204

49. Blaschke AJ, Staley K, Chun J. Widespread programmed cell death in proliferative and postmitotic regions of the fetal cerebral cortex. Development (1996) 122(4):1165-74.

50. Allene C, Cossart R. Early NMDA receptor-driven waves of activity in the developing neocortex: physiological or pathological network oscillations? J Physiol (2010) 588(Pt 1):83-91. doi: 10.1113/jphysiol. 2009.178798

51. Murase S, Owens DF, McKay RD. In the newborn hippocampus, neurotrophin-dependent survival requires spontaneous activity and integrin signaling. J Neurosci (2011) 31(21):7791-800. doi: 10.1523/ JNEUROSCI.0202-11.2011

52. Blanquie O, Kilb W, Sinning A, Luhmann HJ. Homeostatic interplay between electrical activity and neuronal apoptosis in the developing neocortex. Neuroscience (2017) 358:190-200. doi: 10.1016/j.neuroscience.2017.06.030

53. Frohlich F, McCormick DA. Endogenous electric fields may guide neocortical network activity. Neuron (2010) 67(1):129-43. doi: 10.1016/ j.neuron.2010.06.005

54. Spitzer NC. Electrical activity in early neuronal development. Nature (2006) 444(7120):707-12. doi: 10.1038/nature05300

55. Luhmann HJ, Sinning A, Yang JW, Reyes-Puerta V, Stuttgen MC, Kirischuk S, et al. Spontaneous Neuronal Activity in Developing Neocortical Networks: From Single Cells to Large-Scale Interactions. Front Neural Circuits (2016) 10:40. doi: 10.3389/fncir.2016.00040

56. Khazipov R, Luhmann HJ. Early patterns of electrical activity in the developing cerebral cortex of humans and rodents. Trends Neurosci (2006) 29(7):414-8. doi: 10.1016/j.tins.2006.05.007 
57. Luhmann HJ, Khazipov R. Neuronal activity patterns in the developing barrel cortex. Neuroscience (2018) 368:256-67. doi: 10.1016/j.neuroscience.2017.05.025

58. Linaro D, Vermaercke B, Iwata R, Ramaswamy A, Libé-Philippot B, Boubakar L, et al. Xenotransplanted Human Cortical Neurons Reveal Species-Specific Development and Functional Integration into Mouse Visual Circuits. Neuron (2019) 104(5):972-86.e6. doi: 10.1016/j.neuron.2019.10.002

59. Real R, Peter M, Trabalza A, Khan S, Smith MA, Dopp J, et al. In vivo modeling of human neuron dynamics and Down syndrome. Science (2018) 362(6416):eaau1810. doi: 10.1126/science.aau1810

60. Trujillo CA, Gao R, Negraes PD, Gu J, Buchanan J, Preissl S, et al. Complex Oscillatory Waves Emerging from Cortical Organoids Model Early Human Brain Network Development. Cell Stem Cell (2019) 25(4):558-69.e7. doi: 10.1016/j.stem.2019.08.002

61. Wilson DE, Smith GB, Jacob AL, Walker T, Dimidschstein J, Fishell G, et al. GABAergic Neurons in Ferret Visual Cortex Participate in Functionally Specific Networks. Neuron (2017) 93(5):1058-65. doi: 10.1016/j.neuron.2017.02.035

62. Najafi F, Elsayed GF, Cao R, Pnevmatikakis E, Latham PE, Cunningham JP, et al. Excitatory and Inhibitory Subnetworks Are Equally Selective during Decision-Making and Emerge Simultaneously during Learning. Neuron (2020) 105(1):165-79. doi: 10.1016/j.neuron.2019.09.045

63. Wong FK, Bercsenyi K, Sreenivasan V, Portalés A, Fernández-Otero M, Marín O. Pyramidal cell regulation of interneuron survival sculpts cortical networks. Nature (2018) 557(7707):668-73. doi: 10.1038/s41586-018-0139-6

64. Duan ZRS, Che A, Chu P, Modol L, Bollmann Y, Babij R, et al. GABAergic Restriction of Network Dynamics Regulates Interneuron Survival in the Developing Cortex. Neuron (2020) 105(1):75-92.e5. doi: 10.1016/ j.neuron.2019.10.008

65. Wu Q, Maniatis T. A striking organization of a large family of human neural cadherin-like cell adhesion genes. Cell (1999) 97(6):779-90. doi: 10.1016/ S0092-8674(00)80789-8

66. Mancia Leon WR, Spatazza J, Rakela B, Chatterjee A, Pande V, Maniatis T, et al. Clustered gamma-protocadherins regulate cortical interneuron programmed cell death. eLife (2020) 9:e55374. doi: 10.7554/eLife.55374

67. Guerrini R, Dobyns WB. Malformations of cortical development: clinical features and genetic causes. Lancet Neurol (2014) 13(7):710-26. doi: 10.1016/S1474-4422(14)70040-7

68. Ben-Ari Y, Holmes GL. Effects of seizures on developmental processes in the immature brain. Lancet Neurol (2006) 5(12):1055-63. doi: 10.1016/S14744422(06)70626-3

69. Rakhade SN, Jensen FE. Epileptogenesis in the immature brain: emerging mechanisms. Nat Rev Neurol (2009) 5(7):380-91. doi: 10.1038/nrneurol.2009.80

70. Marinowic DR, Majolo F, Zanirati GG, Plentz I, Neto EP, Palmini ALF, et al. Analysis of genes involved in cell proliferation, adhesion, and control of apoptosis during embryonic neurogenesis in Induced Pluripotent Stem Cells (iPSCs) from patients with Focal Cortical Dysplasia. Brain Res Bull (2020) 155:112-8. doi: 10.1016/j.brainresbull.2019.11.016

71. D’Mello SR. Regulation of Central Nervous System Development by Class I Histone Deacetylases. Dev Neurosci (2019) 41(3-4):149-65. doi: 10.1159/000505535

72. Luhmann HJ. Models of cortical malformation-Chemical and physical. J Neurosci Methods (2016) 260:62-72. doi: 10.1016/j.jneumeth.2015.03.034

73. Clause A, Kim G, Sonntag M, Weisz CJ, Vetter DE, Rubsamen R, et al. The precise temporal pattern of prehearing spontaneous activity is necessary for tonotopic map refinement. Neuron (2014) 82(4):822-35. doi: 10.1016/ j.neuron.2014.04.001

74. Tiriac A, Smith BE, Feller MB. Light Prior to Eye Opening Promotes Retinal Waves and Eye-Specific Segregation. Neuron (2018) 100(5):1059-65 e4. doi: 10.1016/j.neuron.2018.10.011

75. Bock AS, Binda P, Benson NC, Bridge H, Watkins KE, Fine I. Resting-State Retinotopic Organization in the Absence of Retinal Input and Visual Experience. J Neurosci (2015) 35(36):12366-82. doi: 10.1523/JNEUROSCI. 4715-14.2015

76. van den Hurk J, Van Baelen M, Op de Beeck HP. Development of visual category selectivity in ventral visual cortex does not require visual experience. Proc Natl Acad Sci USA (2017) 114(22):E4501-E10. doi: $10.1073 /$ pnas. 1612862114

77. Masse IO, Guillemette S, Laramee ME, Bronchti G, Boire D. Strain differences of the effect of enucleation and anophthalmia on the size and growth of sensory cortices in mice. Brain Res (2014) 1588:113-26. doi: 10.1016/j.brainres.2014.09.025

78. Rose NR, Bona C. Defining criteria for autoimmune diseases (Witebsky's postulates revisited). Immunol Today (1993) 14(9):426-30. doi: 10.1016/ 0167-5699(93)90244-F

79. Boksa P. On the neurobiology of hallucinations. J Psychiatry Neurosci (2009) 34(4):260-2.

80. Chen P, Ye E, Jin X, Zhu Y, Wang L. Association between Thalamocortical Functional Connectivity Abnormalities and Cognitive Deficits in Schizophrenia. Sci Rep (2019) 9(1):2952. doi: 10.1038/s41598-019-39367-z

81. Curcic-Blake B, Ford JM, Hubl D, Orlov ND, Sommer IE, Waters F, et al. Interaction of language, auditory and memory brain networks in auditory verbal hallucinations. Prog Neurobiol (2017) 148:1-20. doi: 10.1016/ j.pneurobio.2016.11.002

82. Kompus K, Westerhausen R, Hugdahl K. The "paradoxical” engagement of the primary auditory cortex in patients with auditory verbal hallucinations: a meta-analysis of functional neuroimaging studies. Neuropsychologia (2011) 49(12):3361-9. doi: 10.1016/j.neuropsychologia.2011.08.010

83. Lee JH. Disrupted cholinergic modulation can underlie abnormal gamma rhythms in schizophrenia and auditory hallucination. J Comput Neurosci (2017) 43(3):173-87. doi: 10.1007/s10827-017-0666-4

84. Martino M, Magioncalda P, Yu H, Li X, Wang Q, Meng Y, et al. Abnormal Resting-State Connectivity in a Substantia Nigra-Related Striato-ThalamoCortical Network in a Large Sample of First-Episode Drug-Naive Patients With Schizophrenia. Schizophr Bull (2018) 44(2):419-31. doi: 10.1093/ schbul/sbx067

85. Blakemore SJ, Smith J, Steel R, Johnstone CE, Frith CD. The perception of self-produced sensory stimuli in patients with auditory hallucinations and passivity experiences: evidence for a breakdown in self-monitoring. Psychol Med (2000) 30(5):1131-9. doi: 10.1017/S0033291799002676

86. Bais L, Liemburg E, Vercammen A, Bruggeman R, Knegtering H, Aleman A, et al. Effects of low frequency rTMS treatment on brain networks for inner speech in patients with schizophrenia and auditory verbal hallucinations. Prog Neuropsychopharmacol Biol Psychiatry (2017) 78:105-13. doi: 10.1016/ j.pnpbp.2017.04.017

87. Slotema CW, Aleman A, Daskalakis ZJ, Sommer IE. Meta-analysis of repetitive transcranial magnetic stimulation in the treatment of auditory verbal hallucinations: update and effects after one month. Schizophr Res (2012) 142(1-3):40-5. doi: 10.1016/j.schres.2012.08.025

88. Greenhill SD, Juczewski K, de Haan AM, Seaton G, Fox K, Hardingham NR. Neurodevelopment. Adult cortical plasticity depends on an early postnatal critical period. Science (2015) 349(6246):424-7. doi: 10.1126/ science.aaa8481

89. Xu X, Chini M, Bitzenhofer SH, Hanganu-Opatz IL. Transient Knock-Down of Prefrontal DISC1 in Immune-Challenged Mice Causes Abnormal LongRange Coupling and Cognitive Dysfunction throughout Development. J Neurosci (2019) 39(7):1222-35. doi: 10.1523/JNEUROSCI.2170-18.2018

90. Abbas AI, Sundiang MJM, Henoch B, Morton MP, Bolkan SS, Park AJ, et al. Somatostatin Interneurons Facilitate Hippocampal-Prefrontal Synchrony and Prefrontal Spatial Encoding. Neuron (2018) 100(4):926-39 e3. doi: 10.1016/j.neuron.2018.09.029

91. Yoon KJ, Nguyen HN, Ursini G, Zhang F, Kim NS, Wen Z, et al. Modeling a genetic risk for schizophrenia in iPSCs and mice reveals neural stem cell deficits associated with adherens junctions and polarity. Cell Stem Cell (2014) 15(1):79-91. doi: 10.1016/j.stem.2014.05.003

92. Deakin J, Lennox BR, Zandi MS. Antibodies to the N-methyl-D-aspartate receptor and other synaptic proteins in psychosis. Biol Psychiatry (2014) 75 (4):284-91. doi: 10.1016/j.biopsych.2013.07.018

93. Pathmanandavel K, Starling J, Merheb V, Ramanathan S, Sinmaz N, Dale $\mathrm{RC}$, et al. Antibodies to surface dopamine-2 receptor and N-methyl-Daspartate receptor in the first episode of acute psychosis in children. Biol Psychiatry (2015) 77(6):537-47. doi: 10.1016/j.biopsych.2014.07.014

94. Uhlhaas PJ, Singer W, Ursini G. High-frequency oscillations and the neurobiology of schizophrenia. Dialogues Clin Neurosci (2013) 15(3):301-13.

95. Romano M, Fanelli G, Albany CJ, Giganti G, Lombardi G. Past, present, and future of regulatory $\mathrm{T}$ cell therapy in transplantation and autoimmunity. Front Immunol (2019) 10:43. doi: 10.3389/fimmu.2019.00043 
96. Gilani AI, Chohan MO, Inan M, Schobel SA, Chaudhury NH, Paskewitz S, et al. Interneuron precursor transplants in adult hippocampus reverse psychosis-relevant features in a mouse model of hippocampal disinhibition. Proc Natl Acad Sci U S A (2014) 111(20):7450-5. doi: 7450-510.1073/pnas

97. Cascella NG, Schretlen DJ, Sawa AG. Schizophrenia and epilepsy: is there a shared susceptibility? Neurosci Res (2009) 63(4):227-35.

98. Pisetsky DS. The role of innate immunity in the induction of autoimmunity. Autoimmun Rev (2008) 8(1):69-72. doi: 10.1016/j.autrev.2008.07.028

99. Anaya JM. The autoimmune tautology. A summary of evidence. Joint Bone Spine (2017) 84(3):251-3. doi: 10.1016/j.jbspin.2016.11.012

100. Charlson FJ, Ferrari AJ, Santomauro DF, Diminic S, Stockings E, Scott JG, et al. Global Epidemiology and Burden of Schizophrenia: Findings From the Global Burden of Disease Study 2016. Schizophr Bull (2018) 1744(6):1195203. doi: $10.1093 / \mathrm{schbul} / \mathrm{sby} 058$

101. Juraska JM, Willing J. Pubertal onset as a critical transition for neural development and cognition. Brain Res (2017) 1654(Pt B):87-94. doi: 10.1016/j.brainres.2016.04.012

102. Jones I, Chandra PS, Dazzan P, Howard LM. Bipolar disorder, affective psychosis, and schizophrenia in pregnancy and the post-partum period. Lancet (2014) 384(9956):1789-99. doi: 10.1016/S0140-6736(14)61278-2

103. Knuesel I, Chicha L, Britschgi M, Schobel SA, Bodmer M, Hellings JA, et al. Maternal immune activation and abnormal brain development across CNS disorders. Nat Rev Neurol (2014) 10(11):643-60. doi: 10.1038/nrneurol.2014.187

104. Sacco R, Cacci E, Novarino G. Neural stem cells in neuropsychiatric disorders. Curr Opin Neurobiol (2018) 48:131-8. doi: 10.1016/j.conb.2017.12.005

105. Rajarajan P, Borrman T, Liao W, Schrode N, Flaherty E, Casino C, et al. Neuronspecific signatures in the chromosomal connectome associated with schizophrenia risk. Science (2018) 362(6420):eaat4311. doi: 10.1126/science.aat4311

106. Avramopoulos D. Recent Advances in the Genetics of Schizophrenia. Mol Neuropsychiatry (2018) 4(1):35-51. doi: 10.1159/000488679

107. Smeland OB, Frei O, Dale AM, Andreassen OA. The polygenic architecture of schizophrenia - rethinking pathogenesis and nosology. Nat Rev Neurol (2020) 16(7):366-79. doi: 10.1038/s41582-020-0364-0

108. Pers TH, Timshel P, Ripke S, Lent S, Sullivan PF, O'Donovan MC, et al. Comprehensive analysis of schizophrenia-associated loci highlights ion channel pathways and biologically plausible candidate causal genes. Hum Mol Genet (2016) 25(6):1247-54. doi: 10.1093/hmg/ddw007

109. Girdhar K, Hoffman GE, Jiang Y, Brown L, Kundakovic M, Hauberg ME, et al. Cell-specific histone modification maps in the human frontal lobe link schizophrenia risk to the neuronal epigenome. Nat Neurosci (2018) 21 (8):1126-36. doi: 10.1038/s41593-018-0187-0

110. Rizzardi LF, Hickey PF, Rodriguez DiBlasi V, Tryggvadottir R, Callahan CM, Idrizi A, et al. Neuronal brain-region-specific DNA methylation and chromatin accessibility are associated with neuropsychiatric trait heritability. Nat Neurosci (2019) 22(2):307-16. doi: 10.1038/s41593-018-0297-8

111. Mendizabal I, Berto S, Usui N, Toriumi K, Chatterjee P, Douglas C, et al. Cell-type specific epigenetic links to schizophrenia risk in brain. Genome Biol (2019) 20:135. doi: 10.1186/s13059-019-1747-7

112. Thion MS, Ginhoux F, Garel S. Microglia and early brain development: An intimate journey. Science (2018) 362(6411):185-9. doi: 10.1126/science.aat0474

113. Jiang NM, Cowan M, Moonah SN, Petri WA Jr. The Impact of Systemic Inflammation on Neurodevelopment. Trends Mol Med (2018) 24(9):794804. doi: 10.1016/j.molmed.2018.06.008

114. Uhlhaas PJ. Dysconnectivity, large-scale networks and neuronal dynamics in schizophrenia. Curr Opin Neurobiol (2013) 23(2):283-90. doi: 10.1016/ j.conb.2012.11.004

115. MacCabe JH, Wicks S, Lofving S, David AS, Berndtsson A, Gustafsson JE, et al. Decline in cognitive performance between ages 13 and 18 years and the risk for psychosis in adulthood: a Swedish longitudinal cohort study in males. JAMA Psychiatry (2013) 70(3):261-70. doi: 10.1001/2013.jamapsychiatry.43
116. Canitano R, Pallagrosi M. Autism Spectrum Disorders and Schizophrenia Spectrum Disorders: Excitation/Inhibition Imbalance and Developmental Trajectories. Front Psychiatry (2017) 8:69. doi: 10.3389/fpsyt.2017.00069

117. Urs NM, Peterson SM, Caron MG. New Concepts in Dopamine D. Biol Psychiatry (2017) 81(1):78-85. doi: 10.1016/j.biopsych.2016.10.011

118. Hoftman GD, Dienel SJ, Bazmi HH, Zhang Y, Chen K, Lewis DA. Altered Gradients of Glutamate and Gamma-Aminobutyric Acid Transcripts in the Cortical Visuospatial Working Memory Network in Schizophrenia. Biol Psychiatry (2018) 83(8):670-9. doi: 10.1016/j.biopsych.2017.11.029

119. Lieberman JA, First MB. Psychotic Disorders. N Engl J Med (2018) 379 (3):270-80. doi: 10.1056/NEJMra1801490

120. Orhan F, Fatouros-Bergman H, Goiny M, Malmqvist A, Piehl FKarolinska Schizophrenia Project C, et al. CSF GABA is reduced in first-episode psychosis and associates to symptom severity. Mol Psychiatry (2018) 23 (5):1244-50. doi: 10.1038/mp.2017.25

121. de la Fuente-Sandoval C, Reyes-Madrigal F, Mao X, Leon-Ortiz P, Rodriguez-Mayoral O, Solis-Vivanco R, et al. Cortico-Striatal GABAergic and Glutamatergic Dysregulations in Subjects at Ultra-High Risk for Psychosis Investigated with Proton Magnetic Resonance Spectroscopy. Int J Neuropsychopharmacol (2015) 19(3):pyv105. doi: 10.1093/ijnp/pyv105

122. Cassidy CM, Balsam PD, Weinstein JJ, Rosengard RJ, Slifstein M, Daw ND, et al. A Perceptual Inference Mechanism for Hallucinations Linked to Striatal Dopamine. Curr Biol (2018) 28(4):503-14 e4. doi: 10.1016/j.cub.2017.12.059

123. Seshadri SJ, Hoeppner DJ, Tajinda K. Calcium imaging in drug discovery for psychiatric disorders. Front Psychiatry (2020) 11:713. doi: 10.3389/ fpsyt.2020.00713

124. Carrillo-Reid L, Han S, Yang W, Akrouh A, Yuste R. Controlling Visually Guided Behavior by Holographic Recalling of Cortical Ensembles. Cell (2019) 178(2):447-57.e5. doi: 10.1016/j.cell.2019.05.045

125. Jennings JH, Kim CK, Marshel JH, Raffiee M, Ye L, Quirin S, et al. Interacting neural ensembles in orbitofrontal cortex for social and feeding behaviour. Nature (2019) 565(7741):645-9. doi: 10.1038/s41586-018-0866-8

126. Matzinger P. Tolerance, danger, and the extended family. Annu Rev Immunol (1994) 12:991-1045. doi: 10.1146/annurev.iy.12.040194.005015

127. Pradeu T. Immunology and individuality. Elife (2019) 8:e47384. doi: 10.7554/eLife. 47384

128. Damasio A. El extraño orden de las cosas: La vida, los sentimientos y la creación de las culturas. Barcelona: Spain. Ediciones Destino (2018).

129. Picard F, Friston K. Predictions, perception, and a sense of self. Neurology (2014) 83(12):1112-8. doi: 10.1212/WNL.0000000000000798

130. Park SC, Kim YK. A Novel Bio-Psychosocial-Behavioral Treatment Model of Panic Disorder. Psychiatry Invest (2019) 16(1):4-15. doi: 10.30773/pi.2018. 08.21 .1

131. Strik W, Stegmayer K, Walther S, Dierks T. Systems Neuroscience of Psychosis: Mapping Schizophrenia Symptoms onto Brain Systems. Neuropsychobiology (2017) 75(3):100-16. doi: 10.1159/000485221

132. Tew J. A crisis of meaning: can 'schizophrenia' survive in the 21 st century? Med Humanit (2017) 43(2):111-7. doi: 10.1136/medhum-2016-011077

Conflict of Interest: The authors declare that the research was conducted in the absence of any commercial or financial relationships that could be construed as a potential conflict of interest.

Copyright (c) 2020 Sánchez-Ramón and Faure. This is an open-access article distributed under the terms of the Creative Commons Attribution License (CC BY). The use, distribution or reproduction in other forums is permitted, provided the original author(s) and the copyright owner(s) are credited and that the original publication in this journal is cited, in accordance with accepted academic practice. No use, distribution or reproduction is permitted which does not comply with these terms. 